\title{
Fractal characterization of nanostructured materials
}

\author{
A. N. Kovalenko \\ Ioffe Institute, 26 Politekhnicheskaya, St. Petersburg 194021, Russia \\ ras-kan@mail.ru
}

\begin{abstract}
The article presents a developed gradient-pixel method of fractal analysis and results of multifractal characterization of nano- structured materials with a high proportion of non-autonomous phases obtained from micrographs of their surface chips with high-resolution scanning microscopes. Compared with the black and white binarization option, the gray gradation improves the quality of multifractal analysis of nanostructured materials and expands its capabilities, in particular, the selection of multi-scale composite inclusions in the structure of the material and nano-objects on transparent or opaque basis. Establishing the characteristics of these dependencies permits linking the indicators of structural and phase nonuniformity in the development of new materials with changes in their physicochemical properties. In comparison with the fractal dimension of the Sierpinski carpet as a classic regular monofractal computed on the outlined basis, quite accurately coinciding with the known analytical value, the resulting spectrum of fractal dimensions of the synthesized chemical-catalytic and thermoelectric nanomaterials indicates the multifractal nature of their structural and phase nonuniformity according to the Rényi generalized equation.
\end{abstract}

Keywords: nanostructured materials, SEM micrographs of chips, fractal analysis, gradient-pixel method, spectrum of multifractal dimensions.

Received: 2 January 2019

Revised: 31 January 2019

\section{Introduction}

Studies of various processes for the synthesis of nanostructured materials with significantly different properties and composition from homogeneous model media show that fractal self-organization is a characteristic feature of their formation [1-3]. This causes the invariant metric-statistical self-similarity of multi-scale composite structures with a non-Euclidean (fractional) relationship between the rate of increase in the number of considered elements and the increase in the scale of their consideration [4,5]. This is de facto valid for all technologies and methods for the synthesis of nanomaterials and is determined by the special role of the energy and entropy characteristics of the surface and interfacial layers, the correlation scale of the matter and energy transfer phenomena in different macro, micro and nanostructural blocks of the material, as well as the boundary tolerance factor of their partitioning [6-10]. This is clearly seen in materials with a high content of nanostructured non-autonomous phases of a substance. That is, the phases that are thermodynamically not existing in isolation from its macrovolumes and forming on their surface or interfaces under the conditions of spatial and mass restrictions [11,12]. The high specific surface area of fractal aggregates of different nature explains the possibility of their active participation in condensation processes, sorption processes, chemical reactions of heterogeneous catalysis and inhibition, and other applied areas [13]. Establishing the characteristics of these dependencies allows us to link the indicators of structural and phase nonuniformity in the development of new materials with changes in their physicochemical properties, as well as predicting the effect of the size of the fractal object and the spatial scale of its consideration [14-20].

\section{Methodology of fractal analysis}

The calculation-experimental methods of fractal analysis, including the developed gradient-pixel method of characterization of the structural-phase nonuniformity of nanomaterials, are based on the general idea of Mandelbrot of the difference between the topological Euclidean dimension of such non-smooth objects and the geometric dimension. Mathematically, this manifests itself in the form of the dependence $M \sim \varepsilon^{D}$ between the rate of increase in the number of structural elements $M$ under consideration and the increase in the spatial scale interval $\varepsilon$ of their consideration with a fractional index of fractal dimension $D$. The similar pattern also takes place when considering the occurrence of fractal processes of deterministic chaos in time [21]. Here, the time series of the measured signal $U(\tau)$ is displayed in the phase space of the embedding $U^{\prime}=f(U)$ in the form of an attracting set of unstable infinitely jagged phase trajectories, the so-called strange fractal dimension attractor, characterizing the effective number of degrees of freedom, forming a stochastic signal in a dynamic system. For real fractal objects, the self-similarity manifests itself in a certain scaling interval of natural scales of the considered elements size 
$R_{\min } \leq \varepsilon \leq R_{\max }$, where $R_{\min }$ is associated with the finite size of these elements, and $R_{\max }-$ with the tendency of $D$ to its final limit.

The practical assessment of the fractality of real objects (mass fractals, fractal cluster-aggregates, branched structures, fractal surfaces, fractal curves and fractal time series) is made by mathematical processing of experimental data to measure the abovementioned $\varepsilon, M$ scale-structural parameters. The recognized methods of their "in situ" measurements for various powdered, porous, and composite polycrystalline materials, including nanostructured ones, are invasive mercury porosimetry, gas and liquid pycnometry, low-temperature nitrogen adsorption, calorimetry, inverse gas chromatography, small-angle neutron scattering, X-ray diffraction, transmission and scanning electron microscopy and some others [15,22-24]. Some of these methods are used to characterize not only material structure defects (pores, cracks, liquid, gas and solid inclusions), but also for dispersed systems of a different nature (emulsions, aerosols, etc.). In some cases, the identification of the dynamic characteristics of the processes taking place in time can be carried out simultaneously, for example, a topochemical measurement of the reaction rate [25-29]. It should be noted that the use of the outlined invasive methods is quite laborious, requiring high-precision measuring instruments along with special equipment, and is associated with a number of assumptions about the nature of the contact interaction of the substance being embedded with the material under study. In addition, many of the methods of "in situ" measurements of the fractal dimension were developed for a specific task. The use of radiation scattering methods (X-ray, neutron, light) by the fractal structure is sufficiently correct only in case when all the aggregates are characterized by the same size of primary particles and the same nature of their fractal growth.

One of the very common methods of express experimental study of the fractal nature of real nanoclusters in three-dimensional and two-dimensional space is the analysis of their electron microscopic images based on transmission and scanning electron microscopy. Despite the possibility of some subjective errors of the subsequent interpretation of images and a certain deformation of objects in preparation for the experiment, electron microscopy allows the required variation of the image scale and its spatial resolution. However, identical generally accepted algorithms for processing these images have not yet been developed, nor the general algorithms for processing the experimental data on the dynamics of fractal processes in real time. In all cases, the fractal indicator $D$ for the measured $M, \varepsilon$ parameters for spatial structures and for time processes of regular monofractal sets can be obtained geometrically in practice. In the first case, it can be done by the slope ratio of the line, approximating the graph of the above dependence $\ln M \sim D \ln \varepsilon$ in logarithmic coordinates as the $\operatorname{limit} D=\lim _{\varepsilon \rightarrow 0}[\ln M / \ln \varepsilon]$. In the second case, the same can be achieved as the slope of the limit $D=\lim _{\varepsilon \rightarrow 0, K \rightarrow \infty}[\ln C(K, \varepsilon) / \ln \varepsilon]$ dependence for the correlation integral (correlation sum) $C(K, \varepsilon)=K^{-2} \sum_{i, j}^{K} \theta\left[r-\rho\left(x_{i} x_{j}\right)\right]$, which characterizes the averaged probability of proximity of fractal processes phase trajectories. Here, $\varepsilon$ is the characteristic scale of consideration of the phase trajectory attractor elements, $\theta$ is the Heaviside function, $\left[r-\rho\left(x_{i} x_{j}\right)\right]$ is the distance function in the $n$-dimensional phase space, $K$ is number points of the considered attractor in the correlation. The classical mathematical examples of the fractal analysis of ordered geometric models serve as illustrations of this approach: the infinitely jagged Koch curve (or snowflake) having no derivative in any point, the Cantor set or Cantor dust, the Sierpinski carpet, the Menger sponge, the Minkowski sausage. Their fractals are determined analytically and are respectively $D=\ln 4 / \ln 3=1.2618 \ldots ; D=\ln 2 / \ln 3=0.6309 \ldots ; D=\ln 8 / \ln 3=1.8928 \ldots ; D=\ln 20 / \ln 3=$ $2.7268 \ldots ; D=\lim _{\varepsilon \rightarrow 0}[\ln (3 / 2 \sqrt{\varepsilon}) / \ln \varepsilon]=1 / 2$. A rare exception to the definition of fractals as objects with noninteger dimensions is the three-dimensional analogue model of the Sierpinski triangle with $D=\ln 4 / \ln 2=2$, the Peano and Hilbert plane curves with $D=2$, the Fournier Universe with $D=1$, and some others [30-32]. The difference between these examples reflects the fact of non-compactness ("friability") of the fractal in the first case and the dense filling of the space it occupies in the second.

However, it should be noted that for an adequate description of the real unordered (nonuniform) na- tural fractals and many irregular model structures, using only one single value of the fractal dimension $D$ is not enough. Along with the metric characteristics, it is necessary to determine their statistical properties, reflected by the full spectrum of fractal dimensions using the multifractal formalism [32]. The Rényi generalized dimensional equation [33] is its basis:

$$
D_{R q}=\lim _{\varepsilon \rightarrow 0} \lim _{\tau \rightarrow 0} \lim _{m \rightarrow \infty}\left[\frac{1}{q-1} \frac{\ln Z(q, \varepsilon)}{\ln (\varepsilon)}\right]
$$


Here, $I_{R q}(p, q)=[\ln Z(q, \varepsilon)] /(q-1)$ is the Rényi generalized entropy of order $q ; Z(q, \varepsilon)=\sum_{i=1}^{N(\varepsilon)} p_{i}^{q}(\varepsilon)$ is the generalized statistical sum; $N(\varepsilon)$ is the minimum number of the "measuring" cubes with edge $\varepsilon$ required to cover the attractor in $n$-dimensional phase space of embedding; $p_{i}(\varepsilon)$ is the probability of visiting the $i$-th cube by the phase trajectory in this phase space of embedding; $m$ is the number of points used to estimate the dimension (or the number of measurements at time intervals $\tau$ ). The well-known expressions for the Kolmogorov-Sinai entropy and the Kolmogorov-Hausdorff fractal dimension $(q=0)$, the Shannon-Gibbs entropy and the corresponding information dimension $(q=1)$, the correlation entropy and the correlation dimension $(q=2)$ are special cases of this equation with various values of $q$. Since the Rényi dimension is a steadily decreasing function of $q$, in order to display the attractor for larger $q$, a smaller dimension of the embedding space is required. An approximate analog of the Hausdorff dimension is the Minkowski dimension $D=\lim _{\varepsilon \rightarrow 0}[\ln M(\varepsilon) / \ln \varepsilon]$, which coincides with the Hausdorff dimension for the main classical fractals and many applied problems, but has a more efficient calculation algorithm.

For mathematical processing of the dynamic characteristics of the proceeding fractal processes with a limited sequence of the measured signal time series, the Takens theorem is used. According to it, the restoration of the missing coordinates of the attractor trajectories is carried out by using the data from the same series, taken with some delay [34].

\section{Software implementation of the methodology}

The main part of the research published on this base refers to the well-known software packages of fractal time series processing: the FracLab module of the MatLab package, the CDA program (by J. C. Sprott, G. Rowlands), the RQA program (by C. J. Webber, J. P. Zbilut), the TISEAN package (by R. Hegger, H. Kantz, T. Schreiber), the Fractan 4.4 package (by V. Sychev), the CorDimension application (Copyright $\AA$ AIRES), etc. They were briefly reviewed in paper [35]. Less work is devoted to the software implementation of multifractal processing of disordered structures of materials (the MFDrom program by G.V. Vstovsky, the multifractal image analysis program by K. A. Kichigina, A. I. Degtyarev, D. M. Karavaev, etc. $[15,36]$. Since fractal dimension is an invariant property with scale transformation, along with the standard method of physical change of the general scale of consideration of the object being analyzed, the method of digital zooming of its non-detailed elements (the size of "pixels") forming a grid of the complete bitmap image can be used. At the same time, each pixel can be characterized only by one specific color or brightness, and also transparency. The latter possibility has not yet been taken into account. In the above-mentioned papers, either the change in the overall scale of the analyzed object was used, or a variant of black and white binarization of its pixel photographic images. To remove the limitations of this approach in the presence of different-scale composite inclusions in the structure of the material, the grid method of digital gradient pixelation of its micrographs by resizable cells is used as a method for changing the scale of consideration of details of the nanostructured nonuniformity in the algorithm developed below. As a method for identifying nonuniformities in these cells, including those on transparent or non-transparent basis, the gray level gradation of color and transparency of cell images is used.

The gray gradation, in contrast to the black-and-white one used in the above-mentioned works, improves the quality of the multifractal analysis of nanostructured materials and expands its capabilities, in particular, the selection of multi-scale composite inclusions and nano-objects on transparent or non-transparent basis. For each pixel partitioning, as a measure of the fractal spectrum of structural nonuniformity, the probability $p_{i}(\varepsilon)=n_{i} / N(\varepsilon)$ of the gray color distribution in the cells on the set digital scale of its gradation is used (for example, from 0 to 1 ), for the binary black and white gradation the probability of distribution is 0 or 1 . Here, $n_{i}$ is the fixed degree of color gradation in cell $i ; N(\varepsilon)$ is the total number of cells by size $\varepsilon$. Then, depending on the set size of $\varepsilon_{k}$ cells, the generalized correlation function is estimated (generalized statistical sum) $Z(q, \varepsilon)=\sum_{i=1}^{N(\varepsilon)} p_{i}^{q}(\varepsilon)$ for all cells, characterized by the exponent $q$, which can take a value in the interval $-\infty<q<+\infty$. Then, the spectrum of the generalized Rényi fractal dimensions, which characterize the given distribution, is determined using the relation $D_{q}=\tau(q) /(q-1)$ [32]. Here, the function $\tau(q)=(q-1) D_{q}=\lim _{\varepsilon \rightarrow 0}[\ln Z(q, \varepsilon) / \ln (\varepsilon)]$ is defined as the slope ratio of the approximate dependence $Z(q, \varepsilon) \approx \varepsilon^{\tau(q)}$ by the least squares method from the size $\varepsilon$ of cells of the pixel division. The function of the multifractal spectrum is determined $f(\alpha)=q \alpha-\tau(q)$, where $\alpha=d \tau / d q=$ $-\lim _{\varepsilon \rightarrow 0}\left(\sum_{i=1}^{N(\varepsilon)} p_{i}^{q} \ln p_{i}\right) /\left(\sum_{i=1}^{N(\varepsilon)} p_{i}^{q} \ln \varepsilon\right)$ is the derivative of the function $\tau(q)$, note that $\alpha=d \tau /\left.d q\right|_{q \rightarrow+\infty}=$ 
$D_{+\infty}=\alpha_{\min }$ and $\alpha=d \tau /\left.d q\right|_{q \rightarrow-\infty}=D_{-\infty}=\alpha_{\max }$ [32]. Furthermore, the multifractal characteristics are found: with $q=0$ the Hausdorff dimension is $D_{0}=-\lim _{\varepsilon \rightarrow 0}[\ln N(\varepsilon) / \ln \varepsilon]$, where $\left(\sum_{i=1}^{N(\varepsilon)} p_{i}^{q=0}(\varepsilon)\right) \approx N(\varepsilon)$; with $q=1$ the information dimension is $D_{1}=\lim _{\varepsilon \rightarrow 0}\left(\sum_{i=1}^{N(\varepsilon)} p_{i} \ln p_{i}\right) /(\ln \varepsilon)$, where $\left(-\sum_{i=1}^{N(\varepsilon)} p_{i} \ln p_{i}\right)=S(\varepsilon)$ is the Shannon entropy; with $q=2$ the correlation dimension is $D_{2}=\lim _{\varepsilon \rightarrow 0}\left(\sum_{i=1}^{N(\varepsilon)} p_{i}^{2}\right) /(\ln \varepsilon)$, where $\left(\sum_{i=1}^{N(\varepsilon)} p_{i}^{2}\right) \approx C(K, \varepsilon)$ is the correlation integral (correlation sum).

The procedure of gradient gray pixelation with dividing the area of the processed image into a square grid with cells of a certain size $\left(\varepsilon_{k} \times \varepsilon_{k}\right)$ can be included in the overall program using the size doubling algorithm $\varepsilon_{k}=2^{n+1}(n=1,2,3,4,5 \ldots)$. This procedure can also be performed using the standard options of the Adobe Photoshop graphics editor [37], the standard PHP function of the ImageMagick command line utility in the Windows environment [38] and other graphics processing tools with the addition of the "alpha channel" program component in them to define the pixel image transparency.

Obvious criteria for the quality of digital "pixelation" are the ratio of the physical area of the processed image to the number of cells used by pixel partitioning, related to the size of the pixel itself, as well as the number of information units used to describe each pixel of the image.

\section{Results and discussion}

As an example, Fig. 1 shows the micrographs of samples of new model compounds with a high proportion of nanostructured non-autonomous phases for original functional materials for catalytic and thermoelectric purposes that were kindly provided by E. A. Tugova. The samples were synthesized at the Ioffe Institute of the Russian Academy of Sciences on the basis of layered oxides of different composition and structure using different technological methods. The micrographs were obtained using high resolution scanning (raster) electron microscopes JSM-5610 LV and CamScan MV2300 Quanta FEI 200 with direct magnification from 5000 to 300000 times and passport resolution of $2.5 \mathrm{~nm}$ for lines, $3.5 \mathrm{~nm}$ for points.

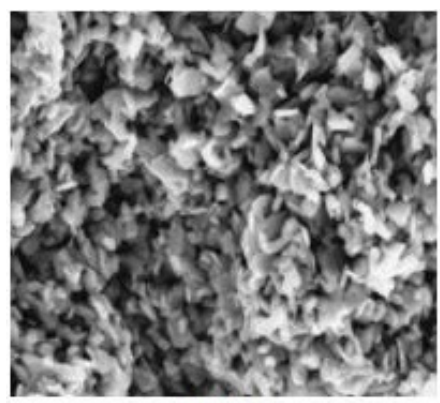

(a)

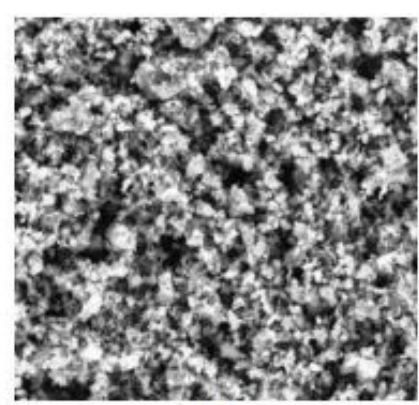

(b)

FIG. 1. Micrographs of the samples of $\mathrm{Ca}_{3} \mathrm{Co}_{4} \mathrm{O}_{9}$ nanocrystalline compounds synthesized by the co-precipitation method with subsequent heat treatment (a) and the gas-flame combustion method of the glycine-nitrate gel precursor (b)

The sample of material according to Fig. 1(a) is characterized by multi-scale composite inclusions in its structure with an average crystallite size of $45 \pm 5 \mathrm{~nm}$ according to X-ray diffraction data. The material was obtained by the method of co-precipitation of cobalt hydroxide $\mathrm{Co}(I I)$ into $\mathrm{CaCO}_{3}$ suspension with $\mathrm{NaOH}$ solution followed by the heat treatment. According to this method, synthesis reactions proceed in a diffusion mode, which is also characteristic of other traditional methods for producing nanodispersed materials based on solid-phase and sol-gel synthesis, hydrothermal and high-temperature processing. The use of the kinetic regime of gas-flame combustion of the glycine-nitrate gel precursor helps solving the problem of sintering the obtained $\mathrm{Ca}_{3} \mathrm{Co}_{4} \mathrm{O}_{9}$ nanopowders with the size of coherent scattering regions of $40 \pm 4 \mathrm{~nm}$ and approximates the properties of thermoelectric nanoceramics on their basis to the properties of single-crystal samples (Fig. 1(b)). 
Examples of the results of partitioning the original electronic images Fig. 1 with square cells with pixel sizes of $4 p, 8 p, 16 p$ and $32 p$, graded in 10 shades of gray, and their comparison with black and white binarization are shown in Fig. 2.

(a)
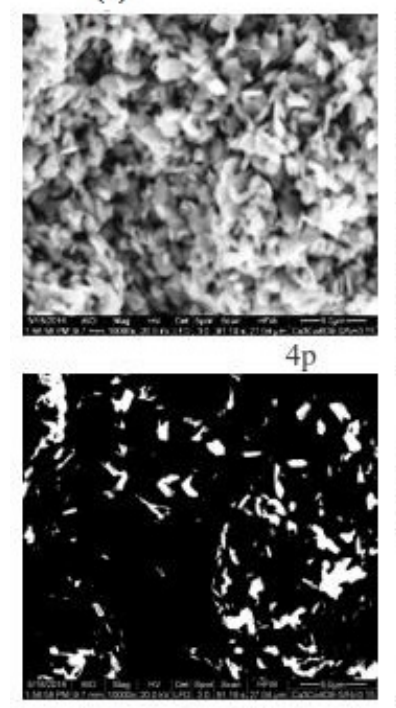

(b)

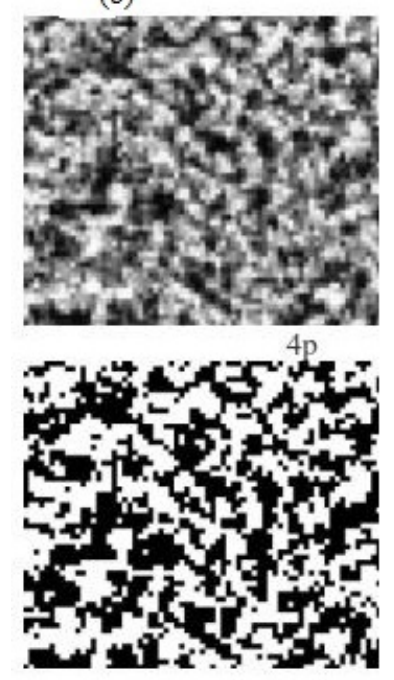

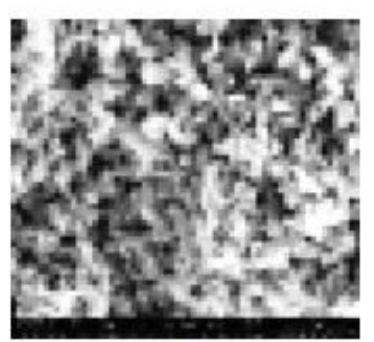

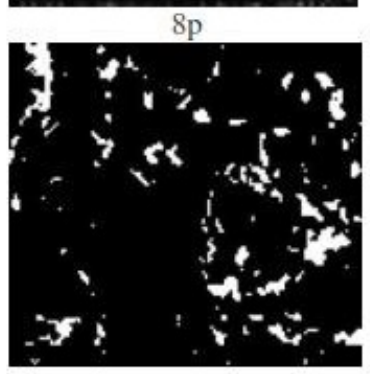

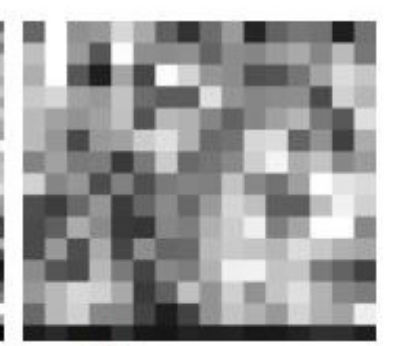

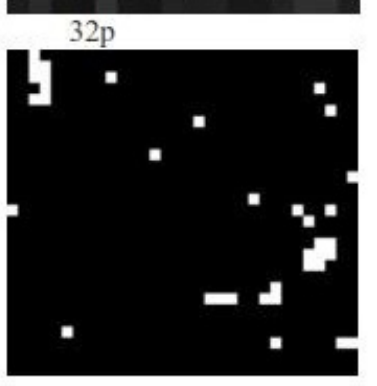

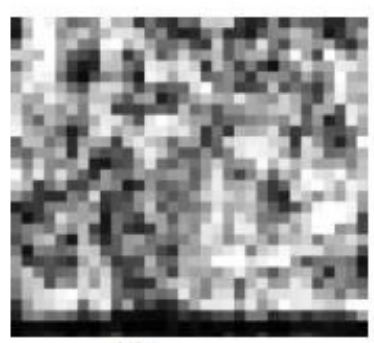
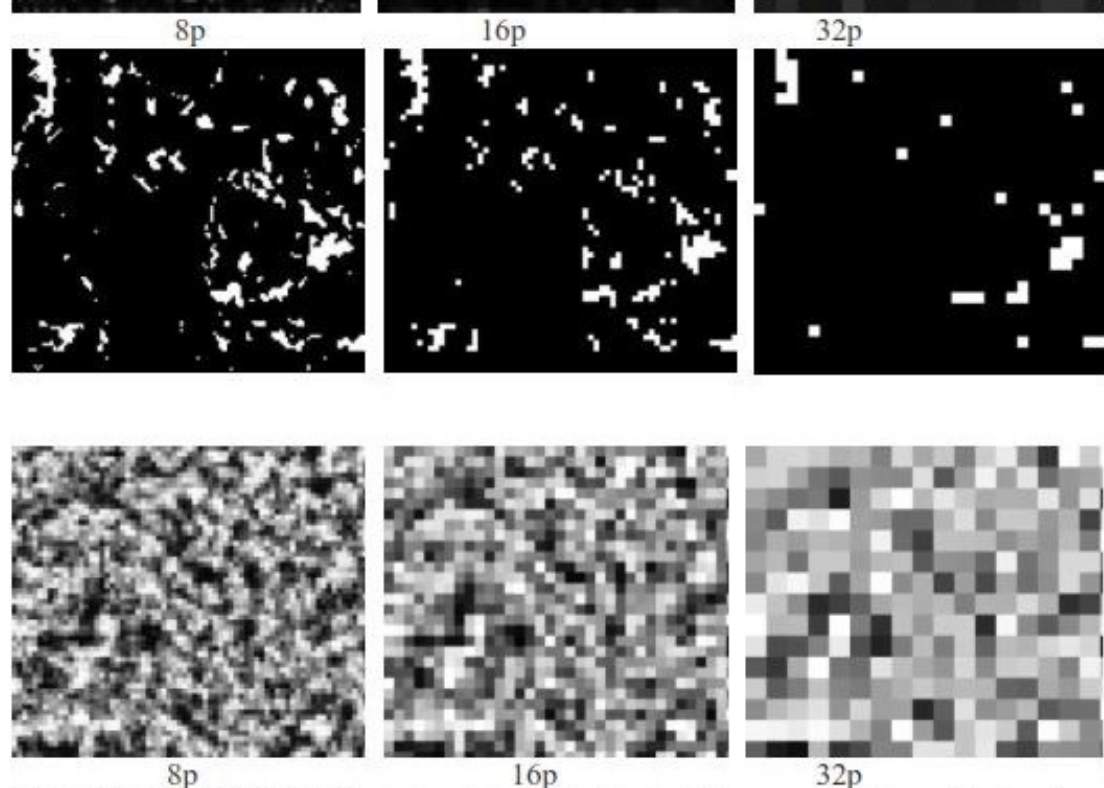
$8 \mathrm{p}$
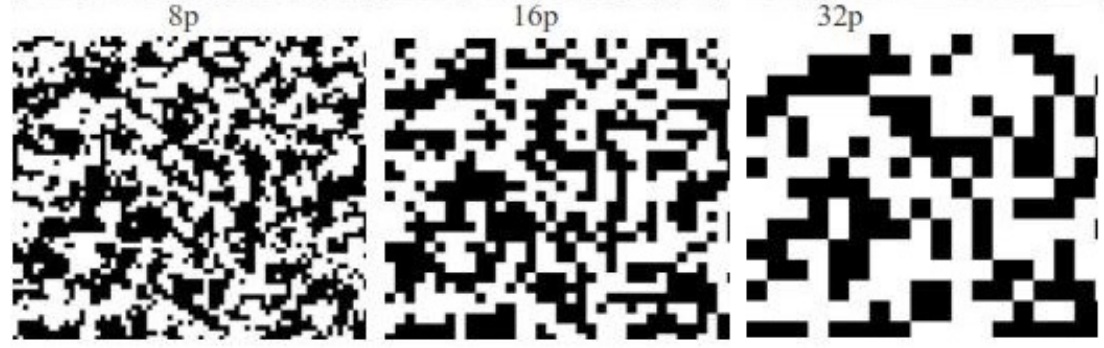

FIG. 2. Comparison of grid partitioning of the source micrographs of synthesized nanocomposite materials samples by cells with pixel sizes $4 p, 8 p, 16 p$ and $32 p$ : (a) for the image of the micrograph Fig. 1(a) in gradation of 10 shades of gray (cells $4 p 8 p, 16 p$ and $32 p$ - top row) and in the black and white binarization variant (cells $4 p 8 p, 16 p$ and $32 p$ - bottom row); (b) - same for the image of the micrograph of Fig. 1(b)

As can be seen, the variant of black and white binarization is associated with a significant loss of the information richness of the analyzed structures as compared to their gray gradation in the gradient pixelation method. The quality of the latter, if necessary, can be improved, for example, up to 256 shades (gradations) of gray color due to the transition to the gray scale widely used in a usual computer representation using 8 bits of information for each pixel of the image. More is possible when using the multibyte full color schemes of $16 / 24 / 30 / 36 / 48$ bits and the transition to the full-color image. In this case, it becomes possible to add the program "alpha channel", which sets the transparency of the image for each pixel $[33,34]$.

For comparison, Fig. 3 shows an example of the gradient pixelation of the classical model image of the Sierpinski carpet as a planar analogue of the regular monofractal Cantor set constructed in the MATLAB-SIMULINK simulation environment [39]. For its construction the method of random iterations was used with the number 
of repeated partitionings of $2^{3}$ with exclusion of the middle thirds by removing the central squares from all the fragments formed by successive division of the sides of the carpet and all its remaining fractions into 3 equal parts.

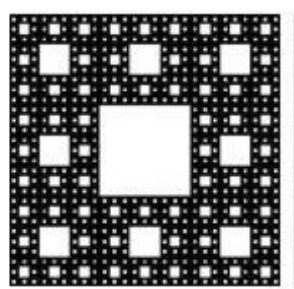

(a)

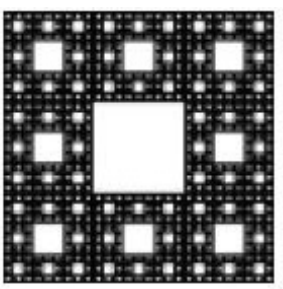

(b)

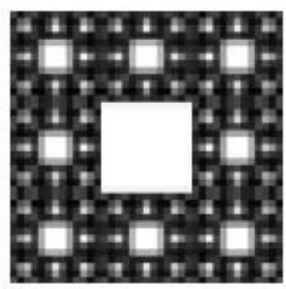

(c)

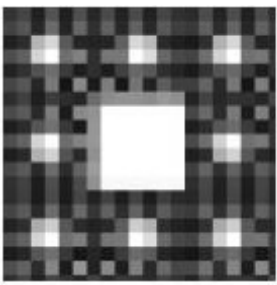

(d)

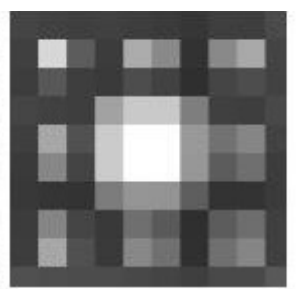

(e)

FIG. 3. The Sierpinski carpet (a), constructed by random iteration with exclusion of middle thirds, and its grid partitionings by gradient cells with pixel sizes of $4 \mathrm{p}(\mathrm{b}), 8 \mathrm{p}$ (c), 16p (g) and $32 \mathrm{p}(\mathrm{d})$

The results of the fractal analysis of the aforementioned model nanostructures based on the developed approach are shown in Fig. 4 in the form of the $D_{q}(q)$ dependence. Thus, Fig. 4(a) shows the fractal dimension of the Sierpinski square carpet model, obtained from its black and white binarized and gradient pixelized images in Fig. 3 and Fig. 4(b) shows the dependence $D_{q}(q)$ obtained from micrographs of the surface chip of a nanocomposite material samples synthesized by different methods on the basis of layered calcium cobaltite $\mathrm{Ca}_{3} \mathrm{Co}_{4} \mathrm{O}_{9} \quad(\mathrm{see}$ Fig. 1). Fig. 4(c) shows the dependence of the function multifractal spectrum $f(\alpha)=q \alpha-\tau(q)$ on the synthesized nanocomposite material from factor $q$ and synthesis method. For calculations, we used a square fragment, maximum in area, with initial resolution from $(1024 \times 768)$ to $(1920 \times 1080)$ pixels with different cell sizes of their "digital" pixelation, graded by 10 shades of gray, examples of which were shown in Fig. 2.

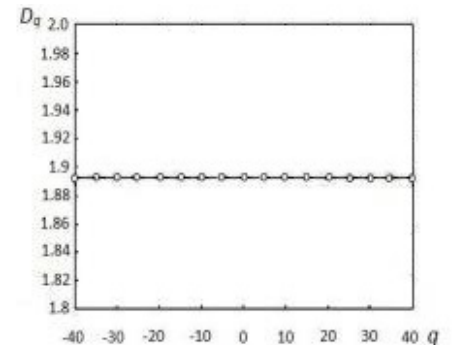

(a)

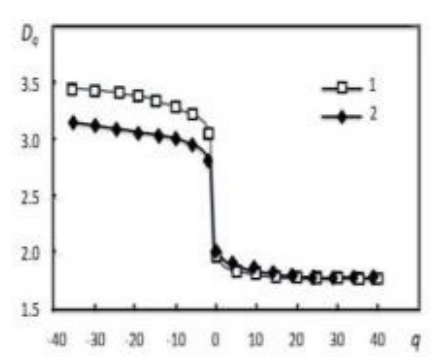

(b)

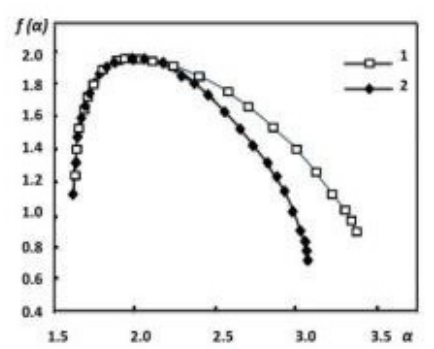

(c)

FIG. 4. Comparison of the calculated fractal dimension from the factor $q$ for the Sierpinski carpet model (a) and for the nanocomposite material (b) synthesized by the co-precipitation method with subsequent heat treatment (1) and the gas-flame combustion method of the glycine-nitrate gel precursor (2) with their function of the multifractal spectrum (c)

As can be seen, the resulting fractal dimension of the Sierpinski carpet as a regular monofractal does not depend on $q$ and with sufficient accuracy coincides with the known analytical value $D=\ln 8 / \ln 3=1.8928 \ldots$. At the same time, the obtained spectrum of the generalized fractal dimensions of the synthesized nanocomposite material depends non-linearly on $q$, indicating the multifractal nature of its structural-phase nonuniformity depending on the synthesis method.

\section{Conclusion}

Fractal self-organization is a characteristic feature of the nanostructured materials formation with significantly different properties and composition from homogeneous model media for virtually all technologies and methods of synthesis. This is manifested in the invariant metric-statistical self-similarity of multi-scale nanocomposite structures with non-Euclidean (fractional) dependence between the rate of increase in the number of elements under consideration and the increase in the scale of their consideration. The value of the fractal dimension of such structures is potentially related to the resulting material properties. This is especially evident when there is a high content of nanostructured non-autonomous phases in the material, that is, the phases that do not exist thermodynamically in isolation from the macrovolumes of the substance and are formed on their surface or 
interfaces under conditions of spatial and mass restrictions. Energy-entropy characteristics and the correlation scale of transfer phenomena in different-sized macro, micro and nanostructural blocks of the material, as well as the boundary tolerance factor can be the key in establishing the relationship between the indicators of structural-phase nonuniformity and the influence of the object's fractal dimensionality on them during the development of new materials with variable physicochemical properties.

Along with in situ measurements of fractal dimensions by invasive methods (mercury porosimetry, gas and liquid pycnometry, low-temperature nitrogen adsorption, calorimetry, inversion gas chromatography) and methods of radiation scattering (X-ray, neutron, light), the analysis of electron microscopic images of nanostructured materials based on the transmission and scanning electron microscopy is very common. Since the fractal dimension is an invariant property in the scale transformation, along with the standard technique of the "physical" changing the overall scale of consideration of the object being analyzed, the method of the "digital" zooming of its non-detailed elements (pixel size) forming a comprehensive raster image can be used. The paper proposes the gray-gradient (if necessary expanded to full-color) pixel method of fractal characterization of nanostructured materials from micrographs of their surface chips using high-resolution scanning (raster) electron microscopes without changing the scale of the processed micrograph itself. Compared with the black and white binarization option, the gray gradation improves the quality of multifractal analysis of nanostructured materials and expands its capabilities, in particular, the selection of multi-scale composite inclusions in the structure of the material and nano-objects on transparent or opaque basis. Obvious quality criteria for such digital "pixelation" are the ratio of the physical area of the image being processed to the number of cells used by pixel partitioning, related to the dimensions of the pixel itself, as well as the number of information units used to describe each pixel of the image.

The obtained results of using this method for characterizing the synthesized samples of new nanostructured materials of chemical-catalytic and thermoelectric purposes with a high proportion of non-autonomous phases in comparison with the classic example of Sierpinsky monofractal model of the carpet indicate the multifractal nature of their structural-phase nonuniformity with a whole range of fractal dimensions according to the generalized Rényi equation.

\section{References}

[1] Kohler M., Fritzsche W. Nanotechnology: An Introduction to Nanostructuring Techniques. Wiley, John \& Sons, Incorporated, 2004, $284 \mathrm{pp}$.

[2] Zhabrev V.A., Kalinnikov V.T., Margolin V.I., Nikolaev A.I., Tupik V.A. Physico-chemical processes of nanoscale objects synthesis. St. Petersburg: Elmor Publishing House, 2012 (in Russian).

[3] Ivanov V.K., Kopitsa G.P., Ivanova O.S., Baranchikov A.Ye., Pranzas K., Grigoriev S.V. Complete inheritance of fractal properties during first-order phase transition. Journal of Physics and Chemistry of Solids, 2014, 75(2), P. 296-299.

[4] Jens Feder. Fractals. Plenum Press. New York and London, 1988, 284 pp.

[5] Mandelbrot B.B. The fractal geometry of nature. San Francisco, Freeman, 1982.

[6] Roldughin V.I. Self-assembly of nanoparticles at interfaces. Russian Chemical Reviews, 2004, 73(2), P. 115-145.

[7] Krasilin A.A., Almjasheva O.V., Gusarov V.V. Effect of the structure of precursors on the formation of nanotubular magnesium hydrosilicate. Inorganic Materials, 2011, 47(10), P. 1111-1115.

[8] Ivanov V.K., Kopitsa G.P., Baranchikov A.E., Grigoriev S.V., Runov V.V., Garamus V. Hydrothermal growth of ceria nanoparticles. Russian Journal of Inorganic Chemistry, 2009, 54(12), P. 1939-1943.

[9] Vasilevskaya A., Almjasheva O.V., Gusarov V.V. Peculiarities of Structural Transformations in Zirconia Nanocrystals. Journal of Nanoparticle Research, 2016, 18(188), P. 1-11.

[10] Korytkova E.N., Pivovarova L.N., Drosdova I.A., Gusarov V.V. Hydrothermal synthesis of nanotubular Co-Mg hydrosilicates with the chrysotile structure. Russ. J. Gen. Chem., 2017, 77(10), P. 1669-1676.

[11] Prigogine I., Defay R. Chemical thermodynamics. Longman Green and Co. London-New York - Toronto, 1954.

[12] Kovalenko A.N., Tugova E.A. Thermodynamics and kinetics of non-autonomous phases formation in nanostructured materials with variable functional properties. Nanosystems: physics, chemistry, mathematics, 2018, 9(5), P. 641-662.

[13] Emets E.P., Novoselova A.E., Poluektov P.P. In situdetermination of the fractal dimensions of aerosol aggregates. Physics-Uspekhi (Advances in Physical Sciences), 1994, 37(9), P. 881-887.

[14] Swapna M.S.,Sankararaman S. Fractal analysis - a surrogate technique for material characterization. Nanosystems: physics, chemistry, mathematics, 2017, 8(6), P. 809-815.

[15] Stryapunina K.A., Makarova L.E., Degtyarev A.I., Karavayev D.M., Matygullina E.V., Sirotenko L.D. The multifractal analysis of the composite material on the basis of thermoexpanded graphite. Izvestia of Samara Scientific Center of the Russian Academy of Sciences, 2014, 16(2), P. 552-556.

[16] Lomanova N.A., Tomkovich M.V., Ugolkov V.L., Volkov M.P., Pleshakov I.V., Panchuk V.V., Semenov V.G. Formation mechanism, thermal and magnetic properties of $\left(\mathrm{Bi}_{1-x} \mathrm{Sr}_{x}\right)_{m+1} \mathrm{Fe}_{m-3} \mathrm{Ti}_{3} \mathrm{O}_{3(m+1)-\delta}(\mathrm{m}=4-7)$ ceramics. Nanosystems: physics, chemistry, mathematics, 2018, 9(5), P. 676-687.

[17] Oksengendler B.L., Ashurov N.R., Maksimov S.E., Uralov I.Z., Karpova O.V. Fractal structures in perovskite-based solar cells. Nanosystems: physics, chemistry, mathematics, 2017, 8(1), P. 92-98.

[18] Ivanov V.K., Baranov A.N., Oleinikov N.N., Tretyakov Yu.D. Fractal surfaces of $\mathrm{ZrO}_{2}$, $\mathrm{WO}_{3}$, and CeO 2 powders. Inorganic Materials, 2002, 38(12), P. 1224-1227. 
[19] Ivanov V.K., Baranov A.N., Oleinikov N.N., Tretyakov Yu.D. Synthesis of iron(III) oxide with controlled surface fractality. Russian Journal of Inorganic Chemistry, 2002, 47(12), P. 1769-1772.

[20] Krivolapova L.I., Kravtsova O.A., Sokolov S.V. Fractal dimension - an estimated quality measure of a surface of metal rolling. Proceedings of Tomsk State University of Control Systems and Radioelectronics, 2015, 1(35), P. 147.

[21] Schuster H.G. Deterministic Chaos. An Introduction. Weinheim, Physik\&Verlag, 1984.

[22] J.Rouquerol, D.Avnir, C.W.Fairbridge et al. Recommendations for the characterisation of porous solids (Technical report). Pure and Applied Chemistry, 1994, 66, P. 1739-1758.

[23] Ivanov V.K., Polezhaeva O.S, Kopitsa G.P., Baranchikov A.E., Tretyakov Yu.D. Fractal structure of ceria nanopowders. Inorganic Materials, 2008, 44(3), P. 272-277.

[24] Yashkin S.N., Svetlov A.A. Study of geometrical heterogeneity of surface of carbon adsorbents with inverse gas chromatography. Russian journal of chemistry and chemical technology, 2009, 52(9), P. 144(70-77).

[25] Ivanov V.K., Oleinikov N.N., Yuri D. Tretyakov Yu.D. Effect of $\mathrm{CeO}_{2}$ surface fractality on the reaction kinetics of ceria with barium nitrate. Russian Journal of Inorganic Chemistry, 2003, 48(4), P. 455-458.

[26] Farin D., Avnir D. Thermal analysis and self-similarity law in particle size distribution of powder samples. Part 4. Thermochimica Acta, 1993, 220, P. 191-201.

[27] Pfeifer P., Avnir D., Farin D. Scaling behavior of surface irregularity in the molecular domain: from absorbtion studies to fractal catalysis. J. Stat. Phys., 1984, 36, P. 699-716.

[28] Schmalzried H. Chemical kinetics of solids. Weinheim, VCH, 1995, P. 433.

[29] Schmidt P.W. Use of scattering to determine the fractal dimension. Fractal Approach to Heterogeneous Chemistry. Ed. D. Avnir. N.Y.: John Wiley\&Sons, 1989.

[30] Crownover R.M. Introduction to Fractals and Chaos. Published by Jones and Bartlett Publishers, Boston and London, 1995.

[31] Falconer K. Fractal Geometry: Mathematical Foundations and Applications, John Wiley \& Sons, New York, 1990.

[32] Bozhokin S.V., Parshin D.A. Fractals and multifractals. Moscow - Izhevsk, Regular and chaotic dynamics, 2001, P. 128.

[33] Renyi A. On a new axiomatic theory of probability. Acta Mathematica Hungaria, 1955, 6, P. $285-335$.

[34] Takens F. Strange Attractors in Turbulence. Dynamical Systems and Turbulence. Lecture Notes in Mathematies. Berlin, Springer-Verlag, 1981, 898, P. 366.

[35] Zakharov A.I., Zagaynov A.I. Multifractal mathematical modeling of processes of chaotic origin. Proceedings of the Mozhaisky Military Space Academy. Mozhaisky MAA, 2015, 648, P. 241.

[36] Vstovsky G.V., Kolmakov A.G., Bunin I.Z. Introduction to Multifractal Parameterization of Material Structures. Izhevsk. Regular and Chaotic Dynamics, 2001. P. 116.

[37] URL: https://www. adobe.com/.

[38] URL: https://www.microsoft.com/.

[39] URL: https://www.mathworks.com/products/matlab.html/. 